BMJ Open Ophthalmology

\title{
Downregulation of interferon- $\gamma$ - induced protein 10 in the tears of patients with Stevens-Johnson syndrome with severe ocular complications in the chronic stage
}

\author{
Mayumi Ueta, ${ }^{1}$ Hiromi Nishigaki, ${ }^{1}$ Chie Sotozono, ${ }^{2}$ Shigeru Kinoshita ${ }^{1}$
}

To cite: Ueta $M$, Nishigaki $H$, Sotozono C, et al. Downregulation of interferon- $\gamma$-induced protein 10 in the tears of patients with Stevens-Johnson syndrome with severe ocular complications in the chronic stage. BMJ Open Ophth 2016;1:e000073.

doi:10.1136/bmjophth-2017000073

Received 30 January 2017 Revised 06 July 2017 Accepted 12 July 2017

\section{CrossMark}

\footnotetext{
${ }^{1}$ Departments of Frontier Medical Science and Technology for Ophthalmology, Kyoto Prefectural University of Medicine, Kyoto, Japan ${ }^{2}$ Department of Ophthalmology, Kyoto Prefectural University of Medicine, Kyoto, Japan
}

Correspondence to Mayumi Ueta; mueta@koto. kpu-m.ac.jp

\section{ABSTRACT}

Objectives Stevens-Johnson syndrome (SJS) and toxic epidermal necrolysis (TEN) are acute inflammatory vesiculobullous reactions of the skin and mucosa such as the ocular surface, oral cavity and genitals. Severe ocular complications (SOC) arise in some patients with SJS/TEN diagnosed by dermatologists. To investigate the pathophysiology of ocular surface inflammation in SJS/TEN with SOC in the chronic stage, we examined cytokines in the tears of patients with ocular surface diseases and healthy controls.

Participants SJS/TEN eyes in the chronic stage $(n>30)$, healthy eyes $(n>20$, controls) and eyes $(n>20)$ from patients with atopic keratoconjunctivitis representing different ocular surface inflammatory disorders.

Primary outcome measures Tear samples were collected on Schirmer's measurement strips. To measure the level of various cytokines in the tears we used BD CBA Flex sets.

Study design An observational study (case-control study).

Results We recorded the level of interleukin (IL)-6, IL8, eotaxin, macrophage inflammatory protein (MIP)- $1 \beta$, RANTES (regulated on activation, normal T cell expressed and secreted), interferon gamma (IFN)- $\gamma$, monocyte chemoattractant protein-1, IFN- $\gamma$-induced protein $10(\mathrm{IP}-10)$ and total $\lg E$. We found that compared with the controls, in SJS/TEN with SOC, IL6 , IL-8, eotaxin and MIP- $1 \beta$ were significantly upregulated while IP-10 was significantly downregulated. Compared with atopic keratoconjunctivitis, IP-10 was significantly downregulated in SJS/TEN with SOC; on the other hand, total IgE was significantly upregulated in atopic keratoconjunctivitis compared with SJS/TEN with SOC.

Conclusions IP-10 in tears may be a biomarker to distinguish between chronic SJS/TEN with SOC and other ocular inflammatory disorders such as atopic keratoconjunctivitis.

\section{Key messages}

It has been reported that interleukin (IL)-6, IL-8 and monocyte chemoattractant protein-1 were dramatically increased in the tear fluid of a patient with Stevens-Johnson syndrome (SJS) in the acute stage; on the other hand, there might be no report on tear cytokines in patients with SJS/TEN (toxic epidermal necrolysis) in the chronic stage. In this study, we found that IFN- $\gamma$-induced protein $10 \quad(\mathrm{IP}-10)$ was significantly downregulated in SJS/TEN with severe ocular complications (SOC) compared with healthy controls and atopic keratoconjunctivitis which was another ocular surface inflammatory disease. IP-10 in tears may be a biomarker to distinguish between chronic SJS/TEN with SOC and other ocular inflammatory disorders such as atopic keratoconjunctivitis.

\section{INTRODUCTION}

Stevens-Johnson syndrome (SJS) is an acute inflammatory vesiculobullous reaction of the skin and mucosa, for example, the ocular surface, oral cavity, respiratory tract and genitals. In patients with extensive skin detachment and a poor prognosis, the condition is called toxic epidermal necrolysis (TEN). Approximately $40 \%$ of patients with SJS/TEN present with severe ocular lesions, for example, severe conjunctivitis with pseudomembrane and ocular surface epithelial defects in the acute stage. ${ }^{1}$

While dermatologists tend to see patients with SJS/TEN only in their acute stage, ophthalmologists usually encounter these patients in the chronic stage. Besides typical ocular surface findings in patients with SJS/ TEN in the chronic stage such as scarring of the lid conjunctiva with severe dry eye, trichiasis and symblepharon, and often 
conjunctival invasion to the cornea, ${ }^{2}$ the ophthalmological diagnosis of SJS/TEN is based on a confirmed history of acute-onset high fever, serious mucocutaneous illness with skin eruptions and involvement of at least two mucosal sites including the ocular surface. In the chronic stage of SJS/TEN with severe ocular complications (SOC), patients may manifest mild ocular surface inflammation. ${ }^{3}$

Elsewhere we reported that among various cytokines, the level of interleukin (IL)-6, IL-8 and monocyte chemoattractant protein-1 (MCP-1), but not of IL-1b, IL-5, eotaxin, interferon (IFN)- $\gamma$ and macrophage inflammatory protein (MIP)- $1 \alpha$, was dramatically increased in the tear fluid and serum of a patient with SJS in the acute stage. ${ }^{4}$ Our search of the literature found no report on tear cytokines in patients with SJS/ TEN in the chronic stage.

Identification of the ocular pathophysiology of chronic SJS/TEN using patient tears, and the detection of differences from other ocular surface diseases, may help elucidate the pathophysiological mechanism(s) underlying the onset of SJS/TEN with SOC. Therefore, we investigated the pathophysiology of ocular surface inflammation in patients with SJS/TEN in the chronic stage by studying various cytokines in their tears. We compared our findings with those made in healthy controls and in patients with other ocular surface inflammatory disorders such as atopic keratoconjunctivitis.

\section{MATERIALS AND METHODS}

Study design

An observational study (case-control study).

\section{Subjects}

Our study protocol was approved by the ethical review board of Kyoto Prefectural University of Medicine; all patients provided prior written informed consent. This study was performed in accordance with the guidelines of the Declaration of Helsinki. We included 40 eyes of patients with SJS/TEN in the chronic stage who had developed SJS/TEN with SOC more than 1 year earlier.

We defined SOC as a condition manifesting pseudomembrane and epithelial defects on the ocular surface (cornea and/or conjunctiva) in the acute stage, ${ }^{5}$ and ocular sequelae such as severe dry eye, trichiasis, symblepharon and conjunctival invasion into the cornea in the chronic stage. ${ }^{3}$

We used tear samples from more than 30 eyes of patients who were seen between 2013 and 2016 by MU in the outpatient clinic of the Department of Ophthalmology, Kyoto Prefectural University of Medicine. The controls were tears from more than 20 eyes of healthy volunteers free of ocular disease. For comparative studies, we collected tears from more than 20 eyes of patients with atopic keratoconjunctivitis.
In patients with $\mathrm{SJS}$ with $\mathrm{SOC}$, we recorded the titre of IL-8, eotaxin, IFN- $\gamma$, MCP-1, total IgE and IFN- $\gamma$-induced protein 10 (IP-10) in 40 eyes, the level of IL- 6 and RANTES (regulated on activation, normal $\mathrm{T}$ cell expressed and secreted) in 36 eyes, and the MIP-1 $\beta$ level in 34 eyes. The level of eotaxin was assayed in 28 eyes of control subjects; IL-6, IL-8, RANTES, IFN- $\gamma$, MCP-1, and total IgE in 27, and IP-10 and MIP-1 $\beta$ in 23 and 22 such eyes, respectively. To determine the titre of IL-6, IL-8, eotaxin, RANTES, IFN- $\gamma$, MCP- 1 and total $\operatorname{IgE}$ in patients with atopic conjunctivitis we used 26 eyes; IP-10 and MIP-1 $\beta$ levels were determined in 22 and 20 such eyes, respectively.

\section{Tear collection}

Tears were collected on Schirmer's measurement strips (Schirmer Tear Production Measuring Strips, Showa Yakuhin Kako, Tokyo, Japan) according to our previously reported method. ${ }^{6}$ They were then immersed in $100 \mu \mathrm{L}$ Tris-buffered saline with Tween 20 (TBST) (DAKO, Japan) for $10 \mathrm{~min}$ at room temperature. Using BD CBA flex sets, we measured the titre of IL-6, IL-8, MCP-1, IFN- $\gamma$, IP-10, MIP-1 $\beta$, eotaxin, RANTES and total IgE in $50 \mu \mathrm{L}$ of TBST containing the tears. The tear volume on the Schirmer strips was calculated at $1 \mu \mathrm{L}$ intervals using a standard curve obtained from 0 to $25 \mu \mathrm{L}$ of distilled water.

\section{Measurement of tear cytokines using CBA kits}

The concentration of cytokines was measured with $\mathrm{BD}$ CBA flex sets and BD human soluble protein master buffer kits according to the manufacturer's instructions (BD Bioscience-PharMingen, San Diego, CA). Briefly, $50 \mu \mathrm{L}$ of mixed capture beads was admixed with $50 \mu \mathrm{L}$ of the provided standards or the tear samples and incubated in the dark for 1 hour at room temperature. Then $50 \mu \mathrm{L}$ of mixed detection reagents was added, the samples were incubated in the dark for 2 hours, washed, centrifuged and resuspended in $300 \mu \mathrm{L}$ of wash buffer. A BD FACS Canto II flow cytometer was calibrated with set-up beads. Data were analysed as recommended by the manufacturer. Concentrations were obtained by interpolating the fluorescence intensity on an at least 7-point dilution standard curve supplied with the kit and calculated with BD FCAP Array software.

Data were expressed as the mean and as individual values and evaluated with Student's t-test and one-way analysis of variance (ANOVA) using Microsoft Excel software. Differences of $\mathrm{p}<0.05$ were considered to be statistically significant.

\section{RESULTS}

We analysed the level of IL-6, IL-8 and MCP-1 because we documented elsewhere ${ }^{4}$ that it was upregulated in tears from patients with SJS/TEN in the acute stage. 

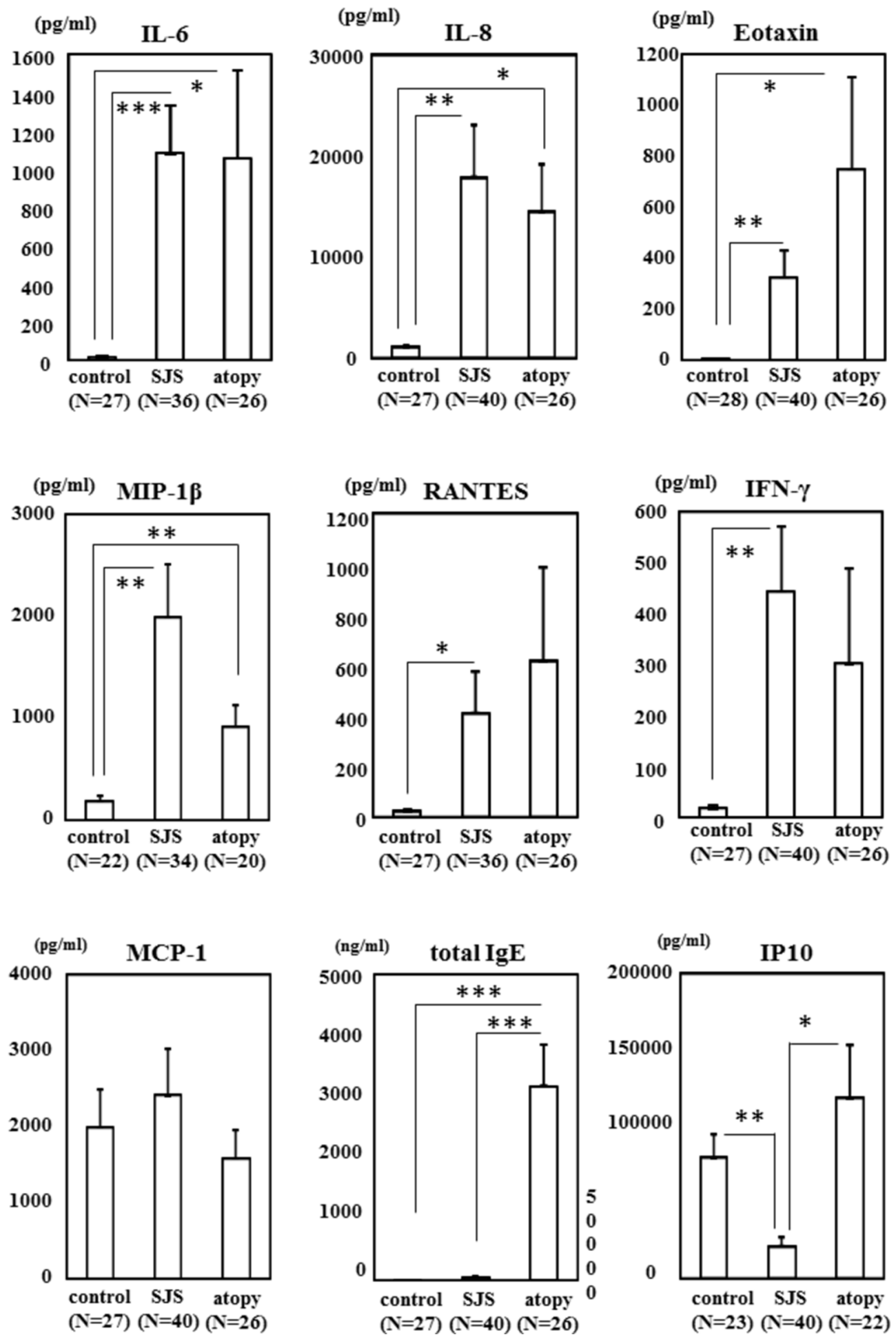

Figure 1 Comparison of cytokine and IgE levels in tears from patients with SJS/TEN with SOC, healthy controls and patients with atopic keratoconjunctivitis. The cytokines were measured in several groups. ${ }^{*} p<0.05,{ }^{* *} p<0.005,{ }^{* * *} p<0.0005$. IFN- $\gamma$, interferon gamma; IL, interleukin; IP-10, IFN- $\gamma$-induced protein 10; MCP-1, monocyte chemoattractant protein-1; MIP-1 $\beta$, macrophage inflammatory protein; N, number of eyes; RANTES, regulated on activation, normal T cell expressed and secreted; SJS, Stevens-Johnson syndrome; SOC, severe ocular complications; TEN, toxic epidermal necrolysis.

We also compared the titre of the Th1 cytokines IFN$\gamma$, IP-10, RANTES and MIP-1 $\beta$, and of eotaxin as another cytokine. Because IgE is a specific marker for allergic diseases, ${ }^{6}$ we also included total $\mathrm{IgE}$ in our study. We confirmed that these cytokines and $\operatorname{IgE}$ could be detected in tears. As our preliminary study using small tear samples to detect the titre of IFN- $\alpha$, IL-4, IL-1 $\beta$, IL-10, IL-12p70 and IL-17F showed that their detection was difficult they were not included in our analyses. 
As shown in figure 1, our comparison of tear samples from patients with SJS/TEN with SOC and the controls revealed significant upregulation of IL- $6(\mathrm{p}<0.0005)$, IL-8 $(\mathrm{p}<0.005)$, eotaxin $(\mathrm{p}<0.005), \operatorname{MIP}-1 \beta(\mathrm{p}<0.005)$, RANTES $(\mathrm{p}<0.05)$ and IFN- $\gamma(\mathrm{p}<0.005)$ in the tears of patients with SJS/TEN with SOC. The level of MCP1 , which we reported to be upregulated in SJS/TEN with SOC in the acute stage, ${ }^{4}$ was similar in patients with SJS/TEN with SOC and the controls. The total IgE level was not significantly different in the patients and the controls. On the other hand, comparison of the IP10 titre showed that it was significantly downregulated in SJS/TEN with SOC $(\mathrm{p}<0.005)$.

When we compared tear samples from patients with SJS/TEN with SOC and atopic keratoconjunctivitis, we found that IP-10 was significantly downregulated in SJS/TEN with SOC $(p<0.05)$; total IgE, on the other hand, was significantly upregulated in atopic keratoconjunctivitis $(\mathrm{p}<0.0005)$. There was no significant difference between patients with SJS/TEN with SOC and patients with atopic keratoconjunctivitis with respect to the level of IL-6, IL-8, eotaxin, MIP- $1 \beta$, RANTES, IFN- $\gamma$ and MCP-1. ANOVA, applied as a multiple comparisons test, revealed that the titre of IL6 , IL-8, eotaxin and MIP- $1 \beta$ was significantly different between the three groups (all $\mathrm{p}<0.05$ ), as was the total IgE titre $(\mathrm{p}<0.000000005)$.

\section{DISCUSSION}

In tears from patients with SJS/TEN with SOC and patients with atopic keratoconjunctivitis, the level of IL-6, IL-8, eotaxin and MIP- $1 \beta$ was upregulated. Their upregulation in both patient groups may be a common phenomenon in the presence of ocular surface inflammatory disorders. On the other hand, IP-10 was downregulated in the tears from patients with chronic SJS/TEN with SOC but not in the presence of atopic keratoconjunctivitis. Others ${ }^{7}$ reported that IP-10 was upregulated in the tears of patients with dry eye. Its downregulation may contribute specifically to the pathogenesis of the SJS with SOC. Like the significant upregulation of total IgE in patients with atopic keratoconjunctivitis, the downregulation of IP-10 in tears may be a biomarker to distinguish chronic SJS/TEN with SOC from other ocular inflammatory disorders. Assay of these serum cytokine levels showed that only RANTES and total IgE could be clearly detected (data not shown). This observation suggests tears are more useful than serum for the detection of biomarkers of ocular surface inflammatory disorders.

Elsewhere we reported that TLR3 polymorphisms are associated with SJS/TEN with SOC, ${ }^{8}$ and that IP-10 was strongly induced by polyI:C, a TLR3 ligand ${ }^{9}$ in conjunctival epithelial cells. We document that in patients with SJS in the chronic stage, the tear IP-10 level was significantly downregulated compared with patients with atopic conjunctivitis and healthy controls.
However, IFN- $\gamma$ was not downregulated although IP-10 and IFN- $\gamma$ are both Th1 cytokines. As IFN- $\gamma$ is primarily produced by immune cells such as lymphocytes, a difference in its dominant producers may account for the different IP-10 and IFN- $\gamma$ levels.

The current study documents that IP-10 regulation is different in patients with SJS/TEN with SOC and patients with other ocular surface inflammatory disorders and suggests strongly that TLR3 contributes to the pathogenesis of SJS/TEN with SOC. To identify the mechanism of ocular surface inflammation of SJS, further investigations and analysis are needed.

Contributors MU: planning, study, sample collection, analysis, paper writing. HN: analysis. CS and SK: paper review.

Funding This work was supported by grants-in-aid from the Ministry of Education, Culture, Sports, Science and Technology of the Japanese government (BioBank Japan Project), and by the JSPS Core-to-Core Program, A: Advanced Research Networks, and also partly supported by grants-in-aid for scientific research from the Japanese Ministry of Health, Labor and Welfare, and a research grant from the Kyoto Foundation for the Promotion of Medical Science and the Intramural Research Fund of Kyoto Prefectural University of Medicine.

Competing interests None declared.

Patient consent Obtained.

Ethics approval Yes

Provenance and peer review Not commissioned; externally peer reviewed.

Open Access This is an Open Access article distributed in accordance with the Creative Commons Attribution Non Commercial (CC BY-NC 4.0) license, which permits others to distribute, remix, adapt, build upon this work noncommercially, and license their derivative works on different terms, provided the original work is properly cited and the use is non-commercial. See: http:// creativecommons.org/licenses/by-nc/4.0/

(C) Article author(s) (or their employer(s) unless otherwise stated in the text of the article) 2016. All rights reserved. No commercial use is permitted unless otherwise expressly granted.

\section{REFERENCES}

1. Sotozono C, Ueta M, Nakatani E, et al. Predictive factors associated with acute ocular involvement in Stevens-Johnson syndrome and toxic epidermal necrolysis. Am J Ophthalmol 2015;160237:e222:228-37.

2. Ueta M, Kinoshita S. Ocular surface inflammation is regulated by innate immunity. Prog Retin Eye Res 2012;31:551-75.

3. Sotozono C, Ang LP, Koizumi N, et al. New grading system for the evaluation of chronic ocular manifestations in patients with StevensJohnson syndrome. Ophthalmology 2007;114:1294-302.

4. Yagi T, Sotozono C, Tanaka M, et al. Cytokine storm arising on the ocular surface in a patient with Stevens-Johnson syndrome. $\mathrm{Br} J$ Ophthalmol 2011;95:1030-1.

5. Sotozono C, Ueta M, Koizumi N, et al. Diagnosis and treatment of Stevens-Johnson syndrome and toxic epidermal necrolysis with ocular complications. Ophthalmology 2009;116:685-90.

6. Ueta M, Shoji J, Sotozono C, et al. Downregulation of IL-8, ECP, and total $\lg \mathrm{E}$ in the tears of patients with atopic keratoconjunctivitis treated with rebamipide eyedrops. Clin Trans/ Allergy 2014;4:40.

7. Enríquez-de-Salamanca A, Castellanos E, Stern ME, et al. Tear cytokine and chemokine analysis and clinical correlations in evaporative-type dry eye disease. Mol Vis 2010;16:862-73.

8. Ueta M, Sotozono C, Inatomi T, et al. Toll-like receptor 3 gene polymorphisms in japanese patients with Stevens-Johnson syndrome. Br J Ophthalmol 2007;91:962-5.

9. Ueta M, Mizushima K, Yokoi N, et al. Gene-expression analysis of polyl:c-stimulated primary human conjunctival epithelial cells. $\mathrm{Br} \mathrm{J}$ Ophthalmol 2010;94:1528-32. 